\title{
Introduction to general analysis $\left(^{*}\right)$
}

\author{
R. G. Lintz (Canada)
}

\begin{abstract}
Summary. - We give here an extension of the concept of derivative to topological spaces, without any metric or linear assumption. For that a new kind of structure has to be introduced, called structure of Gauss space, whose properties ate also interesting in themselves.
\end{abstract}

\section{$\S \mathbf{I}$.}

In this paper we intend to give a generalization of the concept of derivative for general topological spaces. Usually, when we speak about derivative it is always understood that some linear structure is available. However, going back to the origins of Calculus, to the work of NEwToN and LeIBNiz, one realizes that although the concept of linearity is indeed already present in their minds, there are other aspects of derivatives which, Newton, for instance, considers without any need of linear assumptions. That is the case when he looks to the derivative as the quotient of the speeds of two bodies whose movements are related in some way.

The philosophical and historical discussion of these things will be published in a separate paper elsewhere. Here we treat only the mathematical aspect of our ideas. Besides NewTon (7) and Gauss (8) we also owe much to FreCHET (1).

All topological spaces are supoosed to be HAUSDORFF in spite of the fact that many results may be true for more general situations.

1. Let us recall some concepts to begin with. Suppose we have two topological spaces $X$ and $Y$ and suppose $V$ and $V^{\prime}$ are two families of open coverings of $X$ and $Y$, respectively.

Suppose we have: (1) a function

$$
r: \quad V \rightarrow V^{\prime}
$$

(2) for every $\sigma \in V$ a function

$$
f_{\sigma}: \quad \sigma \rightarrow \sigma^{\prime}=r(\sigma) \in V^{\prime} .
$$

(*) Eutrata in Redazione il 6 luglio 1968 . 
Then we say that the collection $\left\{f_{\sigma}\right\}_{\sigma e V}$ is a $g$-function, indicating this by

$$
f: \quad(X, V) \rightarrow\left(Y, V^{\prime}\right)
$$

A $g$-function $f:(X, V) \rightarrow\left(Y, V^{\prime}\right)$ is said to be continuous if $\forall \sigma, \tau \in V$; $\sigma<\tau\left({ }^{1}\right)$, and $\forall A \in \sigma, \forall B \in \tau$ with $B \subset A \Rightarrow f_{\tau}(B) \subset f_{\sigma}(A)$. More details can be found in my papers (2). (6).

2. Definition I. - A standard family of coverings in a topological space $X$ is a family $\mathcal{F}$ of collections $\alpha$ of subsets of $X$ such that:

$I_{1}$ ) Any set $F$ of $\alpha \in \mathscr{F}$ is the closure of a set of $X$, i. e., $F=\bar{A}$, where $A$ is an open set in $X$.

$\left.\mathrm{I}_{2}\right)$ Given $\alpha \in \mathscr{F}$ and any two distinct sets $F_{1}, F_{2} \in \alpha$, then $\stackrel{\circ}{F}_{1} \cap \stackrel{\circ}{F}_{2}=\emptyset\left(^{2}\right)$.

$\left.I_{3}\right)$ Any $\alpha \in \mathscr{F}$ is a covering of $X$.

$\left.\mathrm{I}_{4}\right)$ Given any point $x \in X$ and $\alpha \in \mathcal{F}$ there is a neighbourhood $A$ of $x$ such that $\alpha$ has only a finite number of sets intersecting $A$.

$\mathrm{I}_{5}$ ) Given any open set $A$ of $X$, there is a covering $\alpha \in \mathcal{F}$ such that $\alpha$ has a set $F \subset A$.

$\left.\mathrm{I}_{6}\right)$ Ordered by refinements, $\mathcal{F}$ is a directed set.

Of course, we have to check if all conditions in definition I are independent. Most of them are very easy to see so I restrict myself to one case which could be slightly difficult.

Let $D$ be the family of collections of intervals in $[0,1]$ as shown in the figure below

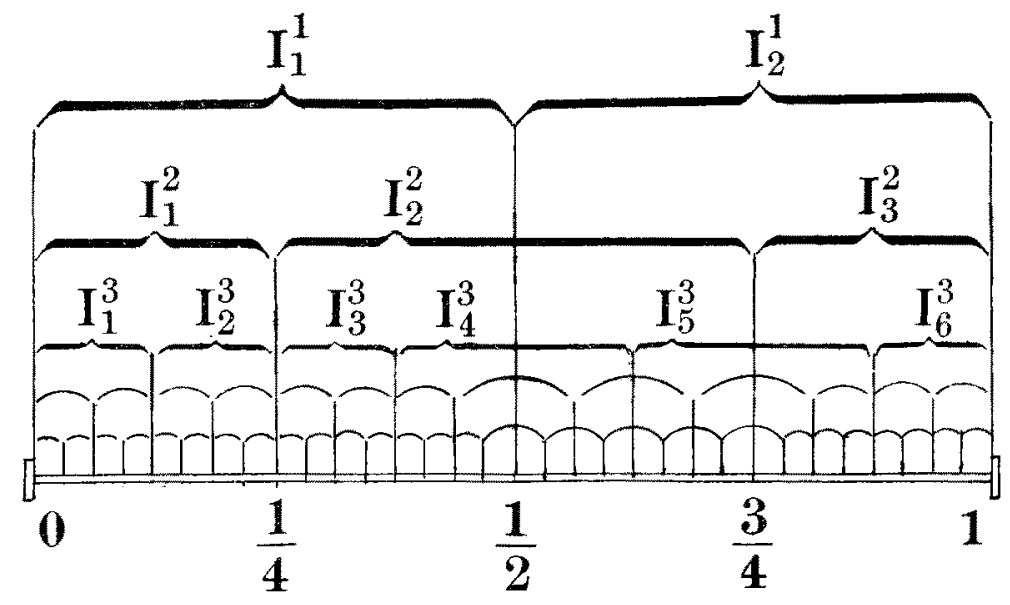

(1) $a<\tau$, means that $\tau$ refines $a$.

(2) $\stackrel{\circ}{F}$ means the interior of $F$ 
The collection $\alpha_{n} \in D$ is made up of the intervals $I_{i}^{n}$. As one sees very easily each $\alpha_{n}$ is not a refinement of any other $\alpha_{m}$ with $n \neq m$, and the family $D$ satisfy all conditions $I_{1}$ to $I_{5}$ of definition $I$, but not, of course, condition $I_{6}$.

However, in the applications it is sometimes useful, having a family $D$ satisfying conditions $I_{1}$ to $I_{5}$ of definition $I$, to be able to construct a family F from $D$ satisfying all conditions of definition $I$, namely, in such a way that $\mathscr{F}$ is a standard family of coverings. This can always be done with the help of a lemma to be proved very soon. Before that, let us introduce some useful notations.

If $\alpha_{1} \ldots \alpha_{n}$ are coverings of $X$, the rotation

$$
\alpha=\alpha_{1} \cap \alpha_{2} \cap \ldots \cap \alpha_{n}=\bigcap_{i=1}^{n} \alpha_{i}
$$

means that $\alpha$ is a covering of $X$ made up of all non empty intersections

$$
A=A_{1} \cap A_{2} \cap \ldots \cap A_{n}
$$

where $A_{i} \in \alpha_{i}, i=1 \ldots n$. The notation

$$
\alpha=\bigcap_{i=1}^{n} \alpha_{i}=\alpha_{1} \cap^{*} \alpha_{2} \cap^{*} \ldots \cap^{*} \alpha_{n}
$$

means that $\alpha$ is a covering of $X$ made up of all non empty intersections

where $A_{i} \in \alpha_{i}, i=1 \ldots n$.

$$
A=\overline{\stackrel{\AA}{A}}_{1} \cap \stackrel{\circ}{A}_{2} \cap \ldots \cap \overline{\check{A}}_{n}
$$

LEMmA A. - Let $D$ be a family of collections of coverings of a topological space $X$, satiafying conditions $I_{1}$ up to $I_{5}$ of definition $I$. Then the family $F$ of all colleotions $\alpha$, with

$$
\alpha=\bigcap_{i=1}^{n} \alpha_{i}
$$

when $\alpha_{i}, i=1 \ldots n$ is a finite set of collections of $D$ is a standard family of coverings of $X$.

Proof. - It is not difficult to check that conditions $I_{1}$ up to $I_{6}$ of definition $I$ are satisfied. As an illustration let us prove, for instance, condition $I_{6}$.

Let $\alpha, \beta \in \mathscr{F}$ arbitrary. We have to find $\gamma \in \mathcal{F}$ refining both $\alpha$ and $\beta$. Take $\gamma=\alpha \cap^{*} \beta$; now any set $H \in \gamma$ is

and

$$
H=\overline{\dot{F}} \cap \bar{G} ; \quad F \in \alpha ; \quad G \in \beta
$$

$$
\begin{aligned}
& \stackrel{\circ}{F}=\stackrel{\circ}{F}_{1} \cap \stackrel{\circ}{F}_{2} \ldots \cap \stackrel{\circ}{F}_{n} ; \quad F_{i} \in \alpha_{i} \in D ; \quad i=1 \ldots n . \\
& \stackrel{\circ}{G}=\stackrel{\circ}{G}_{1} \cap \stackrel{\circ}{G}_{2} \ldots \cap \stackrel{\circ}{G}_{s} ; \quad G_{k} \in \beta_{k} \in D ; \quad k=1 \ldots s
\end{aligned}
$$


Therefore

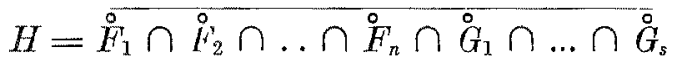

and $\gamma \in \mathcal{F}$. Obvionsly, as $H \subset F \cap G$ we have that $\gamma$ refines both $\alpha$ and $\beta$ and the lemma is proved.

3. Definition II. - A Gause space is a topological space with a stan. dard family of coverings.

The reason for the nomenclature is due to the iact that a standard family of coverings is a generalization of a system of Gauss coordinates in a surface $S$, il we look to the rectangles defined by such a system on $S$. More than that, I show later that a standard family of coverings is also quite adequate to introduce the concept of «curvature» of a topological space, following closely GaUss' ideas, in forthcoming papers.

Theorem 1. - A paracompact space is a GaUss space $\left({ }^{3}\right)$.

Proof. - Suppose $X$ is a paraompact space. Then, there is in $X$ a cofinal family of open coverings $V$, locally finite i. e., each point $x \in X$ has a neighbourhood $V(x)$ intersecting only a finite number of sets of each $\sigma \in V$, where $V(x)$ depends on $\sigma$. We can suppose also that given any open covering $\lambda$ of $X$, there is a $\sigma \in V$ such that for any $A \in \sigma$ there is some $B \in \lambda$ such that $\bar{A} \subset B$.

Now take any point $x \in X$ and consider the intersection of all sets of $\sigma \in V, \sigma$ arbitrary, containing $x$. There is only a finite number of such sets and so we have

$$
x \in A_{1} \cap A_{2} \ldots \cap A_{n}=F_{\sigma}(x) ; \quad A_{i} \in \sigma, i=1,2, \ldots, n .
$$

Consider now

$$
y \in X-\bar{F}_{\sigma}(x)
$$

arbitrary and as before construct the set $F_{\sigma}(y)$.

Suppose now that all points of $X$ are well-ordered and that we bave defined $F_{\sigma}\left(x_{\xi}\right)$ for all $\xi<\eta$, with $\xi$ and $\eta$ ordinal numbers. Let us show how we can define $F_{o}\left(x_{n}\right)$. Take the collection

$$
\Sigma_{\sigma}=\left\{\bar{F}_{\sigma}\left(x_{\xi}\right)\right\}_{\xi<n}
$$

I say this collection is locally finite. Indeed, take any $x \in X$ and $V_{\sigma}(x)$ chosen in such a way that it intersects only a finite number of sets of $\sigma$. Suppose, otherwise, that $V_{\sigma}(x)$ intersects an ininite number of sets of $\Sigma_{\sigma}$.

(3) Actually a student of mine extended this result to much tnore general situations. 
But given any two of these sets, distinct, say $\bar{F}_{\sigma}\left(x_{\xi_{2}}\right)$ and $\bar{F}_{\sigma}\left(x_{\xi_{g}}\right)$, by definition they are obtained by the intersection of different sets from $\sigma$. Therefore, $V_{\sigma}(x)$ intersects also an infinite number of sets of $\sigma$, which is a contradiction. Therefore $\Sigma_{\sigma}$ is locally finite. Observe that as $\Sigma_{\sigma}$ is not a covering it is possible that $V_{\sigma}(x)$ does not intersect any set of $\Sigma_{\sigma}$.

Now, as $\Sigma_{\sigma}$ is locally finite, it is well known that,

$$
\bigcup_{\xi<n} F_{\sigma}\left(x_{\xi}\right)=\bigcup_{\xi<n} \bar{F}_{\sigma}\left(x_{\xi}\right)
$$

and so we can consider the open set in $X$.

$$
X-\bigcup_{\xi<\eta} \bar{F}_{\sigma}\left(x_{\xi}\right)
$$

and if this set is empty we put $\bar{F}_{\sigma}\left(x_{n}\right)=\emptyset$, otherwise we repeat the previous costruction and equally have $\bar{H}_{\sigma}\left(x_{n}\right)$.

Therefore we can suppose that the system $\Sigma_{\sigma}$ covers $X$. Let $\Sigma$ be the family of all collection $\Sigma_{\sigma}$ as $\sigma$ describes $V$.

This collection obviously satisfies conditions $I_{1}, I_{2}, I_{3}$ and $I_{4}$ of defini-

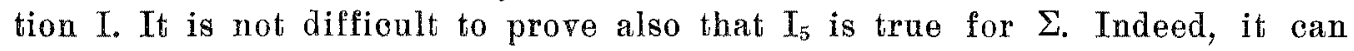
be easily proved that, more generally, given any open covering $\gamma$ of $X$, there is a collection $\Sigma_{\sigma} \in \Sigma$, such that $\Sigma_{\sigma}$ refines $\gamma$.

Now applying lemma $A$, we define a standard family of coverings of $X$ and this completes the proof of the theorem.

Due to this theorem we see that the class of Guass spaces includes important cases, such as paracompact spaces and so if we construct a general analysis for GAUSS spaces this certainly will be indeed «reasonably general», in the sense that it will include important cases.

4. Let $X$ be a Gadss space. This means that in $X$ there is defined a standard family of coverings $\mathscr{F}$. I introduce the notation $(X, \mathscr{F})$ to mean that $X$ is a Gauss space.

Definmion III. - A Gauss transformation from the Gauss space $(X, \mathscr{F})$ into the GaUss space $\left(Y, \mathcal{F}^{\prime}\right)$ is a function $G$,

$$
G: \mathscr{G} \rightarrow \mathscr{F}^{\prime}
$$

compatible with the order by refinement of $\mathscr{F}$ and $\mathfrak{F}^{\prime}$, namely

$$
\forall \alpha, \beta \in \mathcal{F} ; \quad \alpha<\beta \Rightarrow G(\alpha)<G(\beta) .
$$

In the particular case where $X=Y$ and $G$ is one-to-one and onto, we say that $G$ is Gauss transformation of coordinates in $X$. 
Suppose now that $(X, \mathfrak{F})\left({ }^{4}\right)$ and $(Y, \mathscr{F})$ are Gauss spaces and $G$ is a GaUSS transformation from $(X, \mathscr{F})$ into $\left(Y, F^{\prime}\right)$. Let

$$
f:(X, V) \rightarrow\left(Y, V^{\prime}\right)
$$

be a continuous $g$-function with $V$ cofinal in $X$, namely, any open covering in $X$ is refined by some open covering of $V$. Given an $\alpha \in \mathscr{F}$ let us consider for each $x \in X$ an open set $M(x)$ intersecting only a finite number of sets of $\alpha$. The collection of all $M(x)$ is an open covering $\Gamma$ of $X$ and as $V$ is cofinal there is $\sigma \in V$ such that $\Gamma<\sigma$. Therefore, if we take any $A \in \sigma$ there is only a finite number of sets of $\alpha$ intersecting $A$, because $A$ is contained in some $M(x)$. By the definition of $f$, there is a function

$$
r: \quad \nabla \rightarrow V^{\prime}
$$

and so to $\sigma \in V$ we associate $\sigma^{\prime}=r(\sigma)$ and to $A \in \sigma$ we associate $A^{\prime}=f_{\sigma}(A)$.

Let us call $n(A, \sigma \alpha)$ the number of sets of $\alpha$ intersecting $A$. Analogously for $A^{\prime}$, $\sigma^{\prime}$ and $\alpha^{\prime}=G(\alpha)$ we have the number $n\left(A^{\prime}, \sigma^{\prime}, \alpha^{\prime}\right)$, possibly infinite now. Let us define in general

$$
\begin{gathered}
D_{A}(f, \sigma, \alpha)=\infty, \text { if }\left\{\begin{array}{l}
n\left(A^{\prime}, \sigma^{\prime}, \alpha^{\prime}\right) \text { is infinite and } \\
n(A, \sigma, \alpha) \text { is finite }
\end{array}\right. \\
D_{A}(f, \sigma, \alpha)=\frac{n\left(A^{\prime}, \sigma^{\prime}, \alpha^{\prime}\right)}{n(A, \sigma, \alpha)} \text { if both numerator and denominator are finite. } \\
D_{A}(f, \sigma, \alpha)=0, \text { if }\left\{\begin{array}{l}
n\left(A^{\prime}, \sigma^{\prime}, \alpha^{\prime}\right) \text { is finite and } \\
n(A, \sigma, \alpha) \text { is infinite }
\end{array}\right.
\end{gathered}
$$

$D_{A}(f, \sigma, \alpha)$ is not defined if both $n\left(A^{\prime}, \sigma^{\prime}, \alpha^{\prime}\right)$ and $n(A, \sigma, \alpha)$ are infinite.

With these conventions we can define the numbers,

$$
\begin{aligned}
& \bar{D}_{A}(f, \sigma)=\varlimsup_{\alpha \in \mathcal{F}} D_{A}(f, \sigma, \alpha) \\
& D_{A}(f, \sigma)=\lim _{\alpha \in \mathcal{F}} D_{A}(f, \sigma, \alpha),
\end{aligned}
$$

with limits in Moore-SMith sense.

(4) From now on we always suppose that if $\alpha, \beta \in \mathscr{F}$ also $x \Pi^{*} \beta \in \mathscr{F}$. This is possible by Lemma $A$ and is a fundamental hypothesis in what follows. 
We remark that by our previous choice of $\alpha$ these limits have a well defined value, maybe, infinite; recalling the footnote above.

In this way to each $A \in \sigma$ we associate two numbers $\bar{D}_{A}(f, \sigma)$ and $D_{A}(f, \sigma)$. If they are different we associate to $A$ the open interval in the reals $R$, having these numbers as extremities. If they are equal, we associate with $A$ their common value. So, we have a function $D_{\sigma}(f)$,

$$
D_{\circ}(f): \quad \sigma \rightarrow \sigma_{K}
$$

associating to each $A \in \sigma$ an interval or a point in $R$, calling the collection of these by $\sigma_{R}$, namely, $\sigma_{R}$ is a collection of subsets of $R$ containing open intervals and points.

Now, as $\sigma$ changes in $V$ we have a family

$$
\left(D_{\sigma}(f)\right\}_{\sigma \in V_{0}}
$$

of such function, where $\nabla_{0}$ is the family of all coverings $\sigma$ of $V$ such that, any open set $A \in \sigma$ intersect only a finite number of sets of an $\alpha \in \mathcal{F}$.

Therefore we have here something close to a $g$-function and in general we call a special $g$-function the following

$\left.\mathrm{E}_{1}\right)$ Two pairs $(X, V)$ and $\left[R, \nabla_{K}\right]$ where $X$ is a topologieal space and $V$ a family of open coverings of $X ; R$ is the space of real numbers and $V_{R}$ a family of collections $\sigma_{R}$, where each $\sigma_{R}$ is a set of open intervals with non-negative extremities or a set of non-negative real numbers.

$\mathrm{E}_{2}$ ) A function

$$
r_{V}: \quad V \rightarrow V_{R}
$$

$\left.\mathrm{E}_{3}\right)$ For each $\sigma \in V$, a function

$$
f_{\sigma}: \sigma \rightarrow \sigma_{R}
$$

We use a similar notation as for $g$-function, namely,

$$
f:(X, V) \rightarrow\left[R, \nabla_{R}\right]
$$

So, the collection $\left\{\left.D_{\sigma}(f)\right|_{\sigma \in V_{0}}\right.$ defined above is a special $g$-funetion,

$$
D(f): \quad\left(X, V_{0}\right) \rightarrow\left[R, V_{R}\right]
$$

called the g-derivative of $f\left({ }^{5}\right)$.

(5) As $V_{0}$ is cofinal in $V$, to simplify notation, we write always $\nabla$, instead of $V_{\theta}$. 
5. Now we introduce, what one could name, rules of operation with g-derivatives. In a first moment we discuss some composition laws of $g$-functions.

I) Let $f$ and $g$ be $g$-functions,

$$
\begin{aligned}
& f: \quad(X, V) \rightarrow\left(Y, \nabla^{\prime}\right) \\
& g: \quad\left(Y, V^{\prime} \rightarrow \rightarrow\left(Z, \nabla^{\prime \prime}\right)\right.
\end{aligned}
$$

Each of them is defined by the functions

$$
r_{V}: \quad V \rightarrow V^{\prime} \text { and } \quad r_{V^{\prime}}: \quad V^{\prime} \rightarrow V^{\prime \prime}
$$

and the collections of functions

$$
\left\{f_{\sigma}\right\}_{\sigma \in V} \text { and }\left\{g_{\sigma^{\prime}}\right\}_{\sigma^{\prime} \in V^{\prime}}
$$

Let us consider

$$
\tilde{r}_{V}=r_{V^{\prime}} \circ r_{V}
$$

and

$$
h_{\sigma}=g_{\sigma^{\prime}} \circ f_{\sigma}
$$

with $\sigma^{\prime}=r_{V}(\sigma)$ for each $\sigma \in V$. So these define

$$
h:(X, \nabla) \rightarrow\left(Z, \nabla^{\prime \prime}\right)
$$

called the composite of $f$ and $g$, noted by

$$
h=g \circ f .
$$

Clearly this composition is associative and considering the identity $g$-function

$$
i: \quad(X, \nabla) \rightarrow(X, \nabla)
$$

obviously defined, we have a category whose objects are pairs $(X, \nabla)$ and whose morphisms are $g$-fuctions. Moreover, taking into consideration the 
results of (5) we have a smaller eategory $\left(^{6}\right)$ whose objects are pairs $(X, \nabla)$ with $\nabla$ cofinal and whose morphisms are cofinal continuous $g$-functions $f$. There is a functor from this category to the category of Abelian groups and homomorphisms which associate to each pair $(X, \nabla)$ the CECH homology group $H(X)$ of $X$ with coeficients in an Awelian group $G$ and to each morphism $f$ the induced homomorphism $f_{*}$.

II) As I remarked before we cannot compose in general two special $g$-functions. Nevertheless, we can define some useful composition laws with them. Suppose we have two pairs $(X, V)$ and $\left(Y, \nabla^{\prime}\right)$ and a $g$-funotion

$$
h:(X, V) \rightarrow\left(Y, V^{\prime}\right)
$$

given by

$$
s_{V}: \quad V \rightarrow V^{\prime} \text { and }\left(h_{\sigma}\right)_{\sigma \in V} .
$$

Consider two special $g$-functions

$$
\begin{aligned}
& f: \quad(X, \nabla) \rightarrow\left[R, V_{R}\right] \\
& g: \quad\left(Y, \nabla^{\prime}\right) \rightarrow\left[R, \nabla_{R}^{\prime}\right]
\end{aligned}
$$

such that all intervals and numbers in any collection of $V_{R}$ or $\nabla_{R}^{\prime}$ arefinite. These g-functions, as we know, are given by

$$
r_{V}: \quad V \rightarrow V_{R} \text { and }\left\{f_{\sigma}\right\}_{\sigma E V}
$$

and

$$
r_{V^{\prime}}: V^{\prime} \rightarrow V_{R}^{\prime} \text { and }\left(g_{\sigma^{\prime}}\right]_{\sigma^{\prime} \in V^{\prime}}
$$

Now for each $\sigma \in V$ we consider a collection of open intervals and points in $R$ obtained in the following way: take $A \in \sigma$ and consider $f_{\sigma}(A)$ and $g_{\sigma^{\prime}}, h(A)$ $\sigma^{\prime}=s_{V}(\sigma)$. These may be intervals or numbers. The several possibilities are:

(a) $f_{\sigma}(A)=$ interval with extremities $(a, b)$.

$$
g_{\sigma^{\prime} \circ} h_{\sigma}(A)=\text { interval with extremities }\left(a^{\prime}, b^{\prime}\right) \text {. }
$$

(6) This is not exactly a category in the usual way because the compositon of two continuous $g$-functions is not alway continuous. Any way, when the composition is continuous then it is also functorial, i.e.

$$
h=g \cdot f \Rightarrow h_{* *}=g_{*} \cdot f_{*}
$$


The we define

$$
k_{\sigma}(A)=\left(a a^{\prime}, b b^{\prime}\right)
$$

(b) $f_{\sigma}(A)=$ intervaly with extremities $(a, b)$

$$
g_{\sigma^{\prime}} \circ h(A)=a \text { single number } a^{\prime} .
$$

Then we define

$$
k_{a}(A)=\left(\alpha a^{\prime}, b a^{\prime}\right)
$$

(c) $\quad f_{\sigma}(A)=a$ single number $a$

$$
g_{\sigma^{\prime}} \circ h_{\sigma}(A)=a \text { single number } a^{\prime} \text {. }
$$

Then we define

$$
k_{\sigma}(A)=a a^{\prime} .
$$

(d) $f_{\sigma}(A)=a$ single number $a$

$$
g_{\sigma^{\prime}} \circ h_{\sigma}(A)=\text { an interval }\left(a^{\prime}, b^{\prime}\right) \text {. }
$$

We define

$$
k_{\sigma}(A)=\left(\alpha a^{\prime}, a b^{\prime}\right)
$$

In this way, as $A$ changes in $\sigma, k_{\sigma}$ is a function associating to each open set in $\sigma$ an open interval or a number in $R$. Let us call $\sigma_{R}^{\prime \prime}$ this collection. Now, as $\sigma$ changes in $V$ we have a family $V_{R}^{\prime \prime}$ of collections $\sigma_{R}^{\prime \prime}$ and a function

$$
r_{\sigma}^{\prime \prime}: \quad \nabla \rightarrow V_{R}^{\prime \prime}
$$

and a collection of functions

$$
\left\{k_{\sigma}\right\}_{\sigma \in V} .
$$

These define a special $g$-function

$$
k: \quad(X, V) \rightarrow\left[R, V_{R}^{\prime \prime}\right]
$$

which we call the $g$-product of $f$ and $g$ relatively to $h$ indicating

$$
k=f \underset{k}{\times} g
$$


Also we use the notation

$$
V_{R}^{\prime \prime}=V_{R} \underset{\hat{h}}{\times} \nabla_{R}^{\prime}
$$

In a similar way we can define the $g$-sum of $f$ and $g$ relalively $t o h$, by taking addition instead of multiplication in the former definitions. We use the notations

$$
k=f \underset{h}{+} g
$$

and

$$
\nabla_{R}^{\prime \prime}=\nabla_{R}+\nabla_{k}^{\prime}
$$

There is also no difficulty at all to define the product of a special $g$-function $f$ by a real number $\lambda \geq 0$. And we write $\lambda f$ as a matter of notation.

III) Suppose now we have the $g$-functions

$$
\begin{array}{ll}
f: & (X, \nabla) \rightarrow\left[R, \nabla_{R}\right] \\
g: \quad\left(Y, \nabla^{\prime}\right) \rightarrow\left[R, V_{R}^{\prime}\right] \\
h: \quad\left(Z, \nabla^{\prime \prime}\right) \rightarrow\left[R, V_{R}^{\prime \prime}\right] \\
k_{1}: \quad(X, \nabla) \rightarrow\left(Y, \nabla^{\prime}\right) \\
k_{2}: \quad\left(Y, \nabla^{\prime}\right) \rightarrow\left(Z, V^{\prime \prime}\right),
\end{array}
$$

then, by applying definitions, it is easily proved:

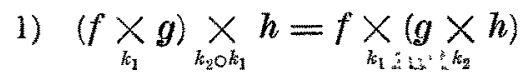

2) $f \underset{k_{1}}{\times}(g+h)=f \underset{k_{k_{1}}}{\times} g+f \underset{k_{2} \circ k_{1}}{\times} h$

where $i$ is the identity $g$-function

$$
i:(X, V) \rightarrow(X, V) .
$$

Usually, we don't write the identity $g$-function $i$ in the expressions as above. Also we remark that in general

$$
f \underset{h}{\times} g \neq g \underset{h^{\prime}}{\times} f
$$

and

$$
f+g \neq g+f
$$


Bat, commutativity holds relatively to identity $g$-function $i$, namely

$$
f \times g=g \times f
$$

and

$$
f+g=g+f
$$

IV) Another relation we can introduce among special $g$-functions is an order relation. Indeed, consider two special $g$-functions

$$
f, g:(X, \nabla) \rightarrow\left[R, V_{R}\right]
$$

and suppose that for arbitrary $\sigma \in V$ and for each $A \in \sigma \in V$ we have

$$
f_{\sigma}(A) \subset g_{\sigma}(A)
$$

Then we say that $f$ is less or equal to $g$ and indicate

$$
f \leq g
$$

It is easily seen that this is a partial ordering. Of course, the same concept can be put for any $g$-functions, but we shall not need this general case by the moment.

V) ChaIN-RuLe. - This is the extension of the classical formula of derivatives of composite functions to the class of $g$-functions, "mutatis mutandis ».

Theorem 2. - Suppose $(X, \mathscr{F}),\left(Y, \mathfrak{F}^{\prime}\right),\left(Z, \mathscr{F}^{\prime \prime}\right)$ are Gauss spaces and suppose we have the $g$-functions

$$
\begin{aligned}
& f: \quad(X, V) \rightarrow\left(Y, V^{\prime}\right) \\
& g: \quad\left(Y, V^{\prime}\right) \rightarrow\left(Z, V^{\prime \prime}\right)
\end{aligned}
$$

Then

$$
D(g \circ f) \leq D(f) \underset{f}{\times} D(g)
$$

Proof. - Suppose $G: \mathscr{F} \rightarrow \mathscr{F}^{\prime}$ and $G^{\prime}: \mathscr{F}^{\prime} \rightarrow \mathscr{F}^{\prime \prime}$ are the Gauss transformations relatively to which we consider the derivatives. Take any $\sigma \in V$ and any $A \in \sigma$. We have

$$
A^{\prime}=f_{\sigma}(A) \text { and } A^{\prime \prime}=g_{\sigma^{\prime}}\left(A^{\prime}\right)
$$


and also

$$
D_{A}(f, \sigma, \alpha)=\frac{n\left(A^{\prime}, \sigma^{\prime}, \alpha^{\prime}\right)}{n(A, \sigma ; \alpha)} \quad \text { and } \quad D_{A^{\prime}}\left(g, \sigma^{\prime}, \alpha^{\prime}\right)=\frac{n\left(A^{\prime \prime}, \sigma^{\prime \prime}, \alpha^{\prime \prime}\right)}{n\left(A^{\prime}, \sigma^{\prime}, \alpha^{\prime}\right)}
$$

where, $\alpha^{\prime}=G(\alpha)$ and $\alpha^{\prime \prime}=G^{\prime}\left(\alpha^{\prime}\right)$, for all $\alpha \in \mathscr{F}$.

So

$$
D_{A}(g \circ f, \sigma, \alpha)=D_{A}(f, \sigma, \alpha) \cdot D_{A^{\prime}}\left(g, \sigma^{\prime}, \alpha^{\prime}\right)
$$

and then

$$
\begin{aligned}
& \varlimsup_{\alpha} D_{A}(g \circ f, \sigma, \alpha) \leq \varlimsup_{\alpha} D_{A}(f, \sigma, \alpha) \cdot \varlimsup_{\alpha} D_{A}\left(g, \sigma^{\prime}, \alpha^{\prime}\right) \\
& \frac{\lim }{\alpha} D_{A}(g \circ f, \sigma, \alpha) \geq \underset{\frac{\lim }{\alpha}}{ } D_{A}(f, \sigma, \alpha) \cdot \frac{\lim }{\alpha} D_{A^{\prime}}\left(g, \sigma^{\prime}, \alpha^{\prime}\right)
\end{aligned}
$$

or

$$
\begin{aligned}
& \bar{D}_{A}(g \circ f, \sigma) \leq \bar{D}_{A}(f, \sigma) \cdot \bar{D}_{A^{\prime}}\left(g, \sigma^{\prime}\right) \\
& \underline{D}_{A}(g \circ f, \sigma) \geq \underline{D}_{A}(f, \sigma) \cdot \underline{D}_{A^{\prime}}\left(g, \sigma^{\prime}\right)
\end{aligned}
$$

and this, of course, means that

$$
D(g \circ f) \leq D(f) \underset{f}{\times} D(g)
$$

ReMark. - When all sequences involved in the proof actually have a limit then we have equality, namely

$$
D(g \circ f)=D(f) \underset{f}{\times} D(g)
$$

VI) Finally we call attention to the formulas

(a) $\quad D(f+g) \leq D(f)+D(g)$

(b) $D(f \times g) \leq D(f) \times g+f \times D(g)$

where

$$
\begin{aligned}
& f: \quad(X, \nabla) \rightarrow\left[R, V_{R}\right] \\
& g: \quad(X, \nabla) \rightarrow\left[R, \nabla_{R}^{\prime}\right]
\end{aligned}
$$

are special $g$-functions. 
6. Derivatives of higher orders. - We arrive now at an important point of our theory. As we shall see, to extend the concept of $g$-derivative to obtain the equivalent of derivatives of higher orders of the ordinary calculus we have to go deeper in the subject and this analysis will be able to show us many new interesting aspects of it.

Up to now it was not quite clear why we restricted ourselves to $g$-deri. vatives of continuous $g$-functions, because apparently this concept has not been used at all. In other words, in a first moment we could obtain the $g$-derivative of any $g$-function, continuous or not.

Let us see that, indeed, we would not obtain, in this case, a reasonable concept of derivative. Suppose

$$
f:(X, \nabla) \rightarrow\left(Y, \nabla^{\prime}\right)
$$

is not continuous. So for some covering $\sigma \in V$ and some open set $A \in \sigma$ we have that for some refinement $\tau$ of $\sigma$ and some $B \in \tau$ with $B \subset A$ it comes out that $f_{\tau}(B) \nsubseteq f_{\sigma}(A)$. That is, the $g$-function $f$ can «scatter around》 open sets contained in $A$ and of course the relations of the speeds of ideal points $P$ in $A$ and $P^{\prime}$ in $A^{\prime}=f_{\sigma}(A)$ would not give a satisfactory picture of the situation, because indeed, for some more accurate measurements of the order of $B$, we have observed that $P^{\prime}$ is outside of $A^{\prime}$. Really to have a nice picture of the situation we should have to take in consideration all possible «scat. terings » of open subsets of $A$, by $f$. But then I don't see any well defined procedure to compute the ratios of speeds of $P$ and $P^{\prime}$. Of course, the idea of taking the union of all scattered open subsets and counting the intersection of the coverings of the standard familv $\mathcal{F}^{\prime}$ in $Y$ with them is not good, be. cause if $B=f_{\tau}(B)$ is "very far from $A^{\prime}$ then really the ideal point $P^{\prime}$ to go from $A^{\prime}$ to $B^{\prime}$ would cover more sets of the coverings of $\mathcal{F}^{\prime}$ than actually taken out by our former computations.

To avoid such troubles with scatterings is the main reason why we restricted ourselves to continuous $g$-functions.

However there is a particular case where we can extend the definition of $g$-derivative to non-continuous $g$-functions: that is the case of special $g$-functions. And it is fortunate we can do that because otherwise I should not know how to define derivatives of higher order.

Suppose

$$
f: \quad(X, V) \rightarrow\left[R, \nabla^{\prime}\right]
$$

is a special $g$-function. Now there are several ways of considering $R$ as a Gauss space, but unless otherwise stated, we shall always consider in $R$ the following standard family of coverings: call $\alpha_{R}^{i}$ the covering of $R$ by closed intervals of length $1 / i$, having only at most the extremities in commun, 
Let $\mathscr{F}_{R}$ be the family of all $\alpha_{R}^{i}, i=1,2, \ldots$. Clearly $\left(R, \mathscr{J}_{R}\right)$ is a GAUSs space, supposing of course, that all intervals in question are defined by starting with the same origin of coordinates in $R$. We refer to $\mathscr{F}_{R}$ as the canonical stan. dard family.

Let $(X, \mathfrak{F})$ be a GaUss space associated with $X$ above and let $G_{R}: \mathscr{F} \rightarrow \mathfrak{F}_{R}$ be a Gauss transformation. Take any $\sigma \in V$ and let $A \in \sigma$ be arbitrary. Consider the collection of intervals or points $f_{\tau}(B)$ in $R$, for all possible refinements $\tau$ of $\sigma$ and all possible $B \in \tau$, with $B \subset A$. Let $A_{R}$ be the smallest possible interval, or eventually a point, containing all sets $f_{\tau}(B)$ above. Consider now the collection $\sigma_{R}^{\prime}$ of all sets $A_{R}$ when $A$ runs in $\sigma$.

Exactly, in a similar way, as we did for the $g$-derivative of a continuous $g$-function we have now the number

$$
D_{A}(f, \sigma, \alpha)=\frac{n\left(A_{R}, \sigma_{R}^{\prime}, \alpha_{R}^{i}\right)}{n(A, \sigma, \alpha)} \quad\left(\alpha_{R}^{i}=G_{R}(\alpha)\right)
$$

Consider now

$$
\begin{aligned}
& \bar{D}_{A}(f, \sigma)=\varlimsup_{\alpha} D(f, \sigma, \alpha)=b_{A} \\
& D_{A}(f, \sigma)=\overline{\lim }_{\alpha} D(f, \sigma, \alpha)=a_{A}
\end{aligned}
$$

Let $\sigma_{R}$ be the collection of all open intervals, eventually points, $\left(a_{A}, b_{A}\right)$ for $A$ running in $\sigma$. Finally let $\nabla_{R}$ be the family of all $\sigma_{R}$. We have then the special $g$-function

$$
D(f): \quad(X, \nabla) \rightarrow\left[R, V_{R}\right]
$$

defined by the family of functions $\left\{D_{a}(f)\right\}_{\sigma \in W^{\prime}}$ where

$$
D_{\sigma}(f): \quad \sigma \rightarrow \sigma_{R}
$$

given by

$$
D_{\sigma}(f)(A)=\left(a_{A}, b_{A}\right)
$$

for all $A \in \sigma \in \nabla$.

The special $g$-function $D(f)$ will be called, as before, the $g$-derivative of $f$.

We remark that in the case of a special $g$-function we are able to control the scattering of open subsets in a well-defined and simple way which is well satisfactory from the intuitive point of view. What is missing in the case of a $g$-function in general is something similar to $A_{R}$ as considered above, 
Another important remark is that now the operation $D$ always remains in the same class, namely, the class of special $g$-functions. This enables us to put the following definition:

Definition IV. - Let $f$ be a continuous $g$-function

$$
f:(X, V) \rightarrow\left(X, V^{\prime}\right) .
$$

The $g$-derivative of $f$ of order $n$, or the $n$-th $g$-derivative of $f$ is given inductively by the formula

$$
D^{n} f=D\left[D^{n-1}(f)\right] ; \quad(n=2,3, \ldots)
$$

\section{\$II.}

1. We begin with the study of the relation between usual derivatives in the sense of classical analysis and $g$-derivatives. To give a satisfactory picture of the situation we need some previous concepts.

Suppose

$$
f: \quad(X, \nabla) \rightarrow\left[R, \nabla^{\prime}\right]
$$

is a special $g$-function. We say that $f$ is continuous if

$$
\forall \sigma, \tau \in V \sigma<\tau \text { and } \forall A \in \sigma, B \in \tau \text {, with } B \subset A \Rightarrow f_{\tau}(B) \subset f_{\sigma}(A)\left({ }^{7}\right) \text {. }
$$

This is exactly the same definition as given before for $g$-functions, extended to the class of special $g$-functions. We remark that if $f_{\circ}(A)=a$, i. e., reduces to a point in $R$, then for any other $B \subset A$, in the condition above, we have $f_{\tau}(B)=a$.

Next one could ask something about the notion of function in the usual sense generated by a special $g$-function.

It is not my intention to discuss in detail this question right now. I content myself in proving a lemma which will be sufficient for our needs at the moment.

Suppose we bave a special $g$-function

$$
f: \quad(X, \nabla) \rightarrow\left[R, V^{\prime}\right]
$$

(7) For later applieations we have to modify this definition but for our needs right now these changes are not necessary. 
and let us assume that every covering $\sigma \in V$ is point-locally finite, namely, given any $x \in X$, there is only a finite number of open sets of $\sigma$ containing $X$.

With this assumption in mind, take any $x \in X$ and any $\sigma \in \nabla$. Then $x$ is contained in the sets $A_{i} \in \sigma,(i=1 \ldots n)$. For each $A_{i}, f_{\sigma}\left(A_{i}\right)$ is an open interval (eventually a point) with extremities $\left(a_{A_{i}}, b_{A_{i}}\right), a_{A_{i}} \leq b_{A_{i}}$. Take the barycenter of all these points putting equal weight in each of them. Let $c_{\sigma}$ be this barycenter.

Let

$$
\bar{\psi}(x)=\varlimsup_{\sigma} c_{\sigma} ; \quad \Psi(x)=\frac{\lim }{\sigma} c_{\sigma}
$$

So for every $x \in X$ we have $\bar{\psi}(x)$ and $\Psi(x)$. Therefore we have two functions

$$
\Psi, \Psi: \quad X \rightarrow R
$$

and they will be called, respectively, the upper and the lower functions generated by $f$.

Lemma B. - Suppose $f:(X, V) \rightarrow\left[R, V^{\prime}\right]$ is a special $g$-function such that:

a) Every $\sigma \in V$ is point-locally finite and $V$ is cofinal in the set of all open coverings of $X$.

b) $\forall x \in X$, and for any number $\varepsilon>0$, there exists $\sigma \in \nabla$, such that $\forall \tau, \sigma<\tau$, implies that the set $\cup f_{\tau}\left(A_{i}\right)$ has diameter less than $\varepsilon$, where the sum is extended all $A_{i} \in \tau$, containing $x$.

Then we have $\bar{\psi}(x)=\psi(x)=\psi(x)$ and $\psi(x)$ is continuous in the usual sense, as a function of $x$, if $f$ is continuous in the sense of special $g$-functions.

Proor. - Suppose $\Psi(x)<\bar{\psi}(x)$ for some $x \in X$. Call $\Psi^{\prime}(x)=a$ and $\bar{\psi}(x)=b$. So there are disjoint neighborhoods $V(a)$ and $V(b)$ of $a$ and $b$ in $R$. To fix the ideas let $\delta$ be the distance between $a$ and $b$, i. $\theta$, ,

$$
\delta=\operatorname{dist}(a, b)
$$

and let $V(a)$ and $V(b)$ be open intervals with centers at $a$ and $b$ respectively and length $2 \delta / 3$.

Now by the definitions of $a$ and $b$, there exist coverings $\sigma_{1}, \sigma_{2} \in \nabla$ such that, for any covering $\tau$ refining $\sigma_{1}$, we have $c_{\tau} \in \nabla(a)$ and $c_{\gamma} \in \nabla(b)$ for $\sigma_{2}<\gamma$. Take now $\varepsilon=\delta / 3$. Then there is $\bar{\sigma} \in V$ such that $\sigma_{1}, \bar{\sigma}_{2}<\sigma$ and if $\nu \in \nabla$, with $\bar{\sigma}<\nu$ we have

$$
\operatorname{diam}\left(\cup f_{\nu}\left(A_{i}\right)\right)<\delta / 3
$$


where the sum is extended to all $A_{i} \in \nu$ containing $x$. This, of course, contradicts condition $b$ ). Therefore, as $\underline{\psi}(x) \leq \bar{\psi}(x)$ we must have

$$
\Psi(x)=\bar{\psi}(x)=\psi(x)
$$

for all $x \in X$.

Now let us see that if $f$ is continuons as special $g$-function then $\psi(x)$ is a continuous function of $x$ in the usual sense. Indeed, let $\nabla(y)$ be an open interval of length $\delta$ and center at $y=\psi(x)$. Using condition $b$ ) again, take $\varepsilon=\delta / 2$ and $\sigma$ with the required properties. Call $A$ the intersection of all open sets of $\sigma$ containing $x$ and take an arbitrary $z \in A$. As $V$ is cofinal there is $\tau \in V$ refining $\sigma$ such that the union of all open sets of $\tau$ containing $z$ is contained in $A$. Now, by the continuity of $f$ we have that $c_{\tau}$ belongs to $V(y)$. But now by the definition of $\psi$, also $\psi(z) \in V(y)$. This completes the proof.

REMARK. - If $f$ were a cofinal $g$-function

$$
f:(X, \nabla) \rightarrow\left(Y, \nabla^{\prime}\right)
$$

where $Y$ is a linear metric space and $V^{\prime}$ cofinal in the family of all open coverings of $Y$ then condition $b$ ) of the lemma is satisfied if $a$ ) is supposed to be true. Now, with identical proof we also arrive at the conclusion that $f$ generates a usual continuous function $\varphi: X \rightarrow Y$. This is related to a former result of mine (see: LrNTz, R. G. (4); p. 99, th. 1).

2. Now we can prove the main result of section $A$.

Theorem 3. - Let $\varphi: R \rightarrow R$ be a real function of real variable defined and differentiable for all real numbers. Let $\mathfrak{F}_{R}$ be the canonical standard family of coverings of $R$ and let $G_{R}: \mathscr{F}_{R} \rightarrow \mathscr{F}_{R}$ be the identical GaUss transformation. Then there exists a continuous $g$-function generating $\varphi$ such that Df generates a function $\psi: R \rightarrow R$, with the property:

$$
\forall x \in R \Rightarrow \psi(x)=\left|\varphi^{\prime}(x)\right|
$$

Proof. - Let $V$ be a cofinal family of coverings $\sigma$ of $R$ of order 2, namely, every point in $R$ belongs to at most two open sets of $\sigma$. Consider also in $\sigma$ the intersections of all these sets and finally suppose that all sets of $\sigma$ are open intervals. Let us also recall that the canonical family $\mathcal{F}_{R}$ of $R$ is made up of a countable family of coverings of $R$ by closed intervals and if $\alpha_{j} \in \xi_{R}$ all intervals of $\alpha_{j}$ are of length $1 / j$. Consider the image $\varphi(A)$ of $A \in \sigma$ in $R$ and construct and open interval $A^{\prime}$ in $R$ in the following way: 
a) if $\varphi(A)$ is an open interval take $A^{\prime}=\varphi(A)$;

b) if $\varphi(A)$ is not an open interval then, as $\varphi$ is continuous, it can only be a closed or semi-closed interval or a point. In the two former cases we define $A^{\prime}$ as the interior of that interval and in the latter case we consider an open interval of length $\overline{l(A)^{2}}$, where $l(A)$ is the length of $A$, containing $\varphi(A)$ as middle point.

So, for each $A \in \sigma$ we associate a well defined open interval in $R$. Complete this collection of open intervals in an arbitrary way to obtain an open covering $\sigma^{\prime}$ of $R$. In this way to each $\sigma \in V$ is associated a well defined $\sigma^{\prime}$ and calling $\nabla^{\prime}$ the family of all such $\sigma^{\prime}$ we have a function,

$$
r: \quad \nabla \rightarrow \nabla^{\prime}
$$

Of course, we have also for each $\sigma \in \nabla$ a function

$$
f_{\sigma}: \quad \sigma \rightarrow \sigma^{\prime}
$$

given by a) and b) above. Therefore, the family of functions $\left(\left.f_{\sigma}\right|_{\sigma \in V}\right.$ defines a $g$-function

$$
f: \quad(R, V) \rightarrow\left(R, \nabla^{\prime}\right)
$$

We are going to prove that $f$ satisfies all our requirements.

1) $f$ is continuous. Immediately from definitions.

2) $f$ generates $\varphi$. It is only sufficient of note that conditions a) and b) of Lemma $B$ are satisfied and so $f$ generates a continuous function $\theta$. Now it is not difficalt to see that $\theta=\varphi$.

3) Df generates a function $\psi$ such that for any $x \in R$ we have $\psi(x)=$ $=\left|\varphi^{\prime}(x)\right|$. Indeed, take any $x \in R$ and a covering $\sigma \in V$. Call $A$ the intersection of all sets of $\sigma$ containing $x$. Of course, by definition of $\sigma, A \in \sigma$. We also have

$$
D f_{\sigma}(A)=\lim _{\alpha_{j}} \frac{n\left(A^{\prime}, \sigma^{\prime}, \alpha_{j}^{\prime}\right)}{n\left(A, \sigma, \alpha_{j}\right)} \quad\left(\text { for } \alpha_{j} \in \mathfrak{F}_{R}\right)
$$

Or

$$
\lim _{\alpha_{j}} \frac{n\left(A^{\prime}, \sigma^{\prime}, \alpha_{j}^{\prime}\right)}{n\left(A, \sigma, \alpha_{j}\right)}=\lim _{j \rightarrow \infty} \frac{\frac{1}{j} n\left(A^{\prime}, \sigma^{\prime}, \alpha_{j}^{\prime}\right)}{\frac{1}{j} n\left(A, \sigma, \alpha_{j}\right)}=\frac{l\left(A^{\prime}\right)}{l(A)}
$$


Note that this formula is true even in the case where $\varphi(A)$ reduces to a point. Therefore, in this case $D f$ is a family of functions whose values are real numbers.

Now let $A, B$, and $O$ the intervals of $\sigma$ containing $x$, not excluding the case of all being equal. We have no more than three by the definition of $\sigma$. Call $a, b$, and $c$, respectively the value of $D f_{\sigma}$ in each of these intervals and let $d$ be the barycenter of $a, b$, and $c$. Now

$$
\psi(x)=\lim _{\sigma \in V} d
$$

which certainly exists because all conditions of lemma $B$ are satisfied. But, as $\varphi$ is differentiable we have, due to the geometrical meaning of $a, b$, and $c$, that

$$
\left|\varphi^{\prime}(x)\right|=\lim _{\sigma \in V} a=\lim _{\sigma \in V} b=\lim _{\sigma \in V} c=\lim _{\sigma \in V} d=\psi(x)
$$

This completes the proof of the theorem.

3. The theorem just proved may be considered as a particular case of a more general hypothesis, namely, it is quite possible that if we consider two differentiable manifolds $M, N$ and a differentiable map $\varphi: M \rightarrow N$, then there is a continuous $g$-function

$$
f: \quad(M, \nabla) \rightarrow\left(N, \nabla^{\prime}\right)
$$

generating $\varphi$ and such that $D f$ generates a function $\psi: M \rightarrow R$ with the property :

$$
x \in M \Rightarrow \psi(x)=|\boldsymbol{J}(x)|
$$

where $J(x)$ is the jacobian associated to $f$, at the point $x$.

This result would render in a certain sense the study of differentiable manifolds as a particular case of the study of derivation of $g$-functions

$$
f: \quad(X, \nabla) \rightarrow\left(Y, V^{\prime}\right)
$$

for $(X, F)$ and $(Y, \mathscr{F})$ Gauss spaces. In this way we generalize the ideas of Gadss around the intrinsic study of manifolds.

In few words, we conclude that a structure of Gauss space in $X$ is exactly what was missing for a generalization of the concept of derivative namely, putting into $X$ a topological structure we have what is needed for a general study of continuity and in addition to this, putting also in $X$ a structure of GaUSS space we have what is needed for a general study of derivatives. 


\section{REFERENCES}

M. FrÉChet

(1) Les Espaces Abstraits, Gauthier-Villars Paris (1951).

R. G. LiNTz

(2) Foundations of a New Theory of Topological Manifolds, Annali di Mat. Pura od Appli. cata. (1965), (IV), 67 pp. $301-348$.

(3) A Generalization of the Concept of Continuous Function and Homeomorphism, Annali di Mat. Pura ed Applicata 67 (1965), (IV), pp. 215-234.

(4) On Relations Betreen Usual Continuous Functions and Generalized Continuous Functions, Annali di Mat. Pura ed Applicata 72 (1906), (IV), pp. 97-101.

(5) Generalized Functions and Cech Homology Theory, Annali di Mat. Pura ed Applicata (1906) 72 (IV) pp. 45-58.

(6) Generalieed Fiber-spaces, Annali di Mat. Pura ed Applicata 76 (1967), (IV), pp, 11-26. I. NewToN

(7) Methodus Fluxionum et Serierum Infinitarum, Cum ejusdem Applicatione ad Curva. rum Geometriam.

C. F. Gauss

(8) Desquisitiones Generales Circa Superficies Curvas. 